\title{
Routing and Dimensioning of Optical Networks under Traffic Growth Models: An Asymptotic Analysis
}

\author{
Tapan Kumar Nayak \\ Dept of Electrical Communication Engineering \\ Indian Institute of Science \\ Bangalore, India 560012
}

\author{
Kumar N. Sivarajan \\ Tejas Networks \\ Khanija Bhavan, 49, Race Course Road \\ Bangalore, india 560001
}

\begin{abstract}
In this paper we consider the problem of routing and dimensioning in a large optical network assuming the traffic is growing over time. In optical networks, traffic is carried through lightpaths and we model the traffic as follows: Lightpaths arrive randomly according to a time-varying Poisson process and hold for a random time with a general distribution. We are interested in dimensioning the links so that the first lightpath request rejection will occur, with high probability, after a specified period of time $T$ and thus the network requires no capacity upgradation in that time period. Here we propose a solution based on the absorption probability-the probability that at least one lightpath request will be rejected in $(0, T)$. Computation of exact absorption probability is possible for a few specific holding time distributions (e.g., exponential) and it requires large computing resources. We propose a method which has low computational complexity to approximate the absorption probability for a general holding time distribution based on an asymptotic analysis and we show that it is quite accurate in the desired range of low absorption probabilities.
\end{abstract}

\section{INTRODUCTION}

Fiber optics and wavelength-division multiplexing are evolving as the technologies that can satisfy the huge bandwidth requirements of the Internet today, providing cheap and reliable high-speed communication over long distances. In this work, we consider that all the nodes are capable of full wavelength conversion which results in improved utilization of the available fiber bandwidth in a WDM network [1]. An optical network with full wavelength conversion capability is equivalent to a conventional circuit switched network. For a given traffic distribution, a routing and dimensioning method routes the traffic on appropriate paths and determines the capacities which must be provided to the links so as to meet the network performance while minimizing the cost. Traditional routing and dimensioning methods were designed based on the blocking probability models assuming the network was in a steady state condition [2], [3]. Such models are not appropriate to handle the explosive growth of bandwidth demand today as the traffic is growing constantly and unpredictably without reaching a steady state. Such a dramatic shift in the environment requires a fundamental shift in the network architectures.

We introduce the concept of absorption probability instead of blocking probability to express the grade of service of a network. The motivation behind the selection of absorption probability and its application for network design are discussed in [4] and [5]. The dimensioning problem based on absorption probability is solved for constant arrival rates and the details of the analytical model can be found in [4]. It shows how the absorption probability model outperforms the blocking probability model for constant rates of traffic arrivals. In [5], the dimensioning

The work of Tapan Kumar Nayak was supported by a fellowship from IBM India Research Lab, New Delhi. problem is solved for time-varying traffic arrivals which is more relevant in the current scenario of rapidly growing traffic in optical networks. But in both cases, the dimensioning problem is solved for an exponential holding time distribution assuming the routes are fixed.

In this paper, we propose a solution for both the routing and dimensioning in a large optical network when the traffic arrivals are time-varying Poisson and the holding time distributions are general based on an asymptotic analysis which we describe later.

\section{SUMMARY OF THE WORK}

In section III, we consider a single link of capacity $N$ and derive an explicit expression for the absorption probability as a function of time for an exponential holding time distribution and propose numerical methods for the solution. For a general holding time distribution, we have no choice but to follow the method of simulation. However, we find that if the capacity $N$ and the arrival rate $\lambda(t)$ increase while their ratio remains constant, the absorption probability function converges to a step function as $N \rightarrow \infty$.

In section IV, we consider the asymptotic condition that both $N$ and $\lambda(t) \rightarrow \infty$ while their ratio remains constant and derive the transition time $T_{0}$ for a general holding time distribution. $T_{0}$ is the time when the absorption probability switches from 0 to 1 under the asymptotic condition. We also propose an approximation method to estimate the absorption probability which is very close to the exact solution in the desired range of low absorption probabilities. The complexity of the approximation method is less and it is also useful for a general holding time distribution.

In section $\mathrm{V}$, we propose the routing and dimensioning method for a large optical network so that it requires no upgradation till a given time $T$. For heavy traffic, least cost paths are the optimum routes and the capacities can be obtained immediately from the asymptotic analysis of the individual links. For light traffic, we also propose a method to determine the link capacities along with the lower and upper bounds for a given value of tolerance absorption probability. The lower and upper bounds will converge for heavy traffic.

In section VI, we present the results of the routing and dimensioning for a moderately large network.

\section{ABSORPTION PROBABILITY OF A SINGLE LINK}

Here we consider a single link network of capacity $N$. We assume the arrivals are Poisson with $\lambda(t)$ as the state-independent arrival rate at time $t$; that is, the probability of $j$ arrivals in a time interval $(0, t)$ is given by $[6]$

$$
\exp \left(-\int_{0}^{t} \lambda(u) \mathrm{d} u\right) \frac{\left[\int_{0}^{t} \lambda(u) \mathrm{d} u\right]^{j}}{j !} .
$$


For an exponential holding time distribution with constant mean $1 / \mu$, the busy wavelength distribution can be modeled by a birth-death process where a state of the Markov chain represents the number of lightpaths in progress. Let $P_{n}(t)$ be the probability of state $n$ at time $t$. Then the relations between the state probabilities at times $t+h$ and $t$ are as following [7]:

$$
\begin{aligned}
& P_{n}(t+h)= P_{n}(t)\{1-\lambda(t) h-n \mu h\}+\lambda(t) h P_{n-1}(t)+ \\
&(n+1) \mu h P_{n+1}(t)+o(h) \quad \text { for } 1 \leq n \leq N \cdot-1,(1) \\
& P_{0}(t+h)= P_{0}(t)\{1-\lambda(t) h\}+\mu h P_{1}(t)+o(h), \\
& P_{N}(t+h)= P_{N}(t)\{1-\lambda(t) h-N \mu h\}+\lambda(t) h P_{N-1}(t)+o(h),(3) \\
& P_{N+1}(t+h)= P_{N+1}(t)+\lambda(t) h P_{N}(t)+o(h) . \\
& \text { Initial Condition: } P_{0}(0)=1, \quad P_{n}(0)=0 \text { for } n>0 .
\end{aligned}
$$

The solution of the absorption probability $P_{N+1}(t)$ for a single link network can be obtained explicitly from (1)-(4) as a function of $t$ using the generating functions of the state probabilities and the corresponding binomial moments. We follow a similar approach to obtain the absorption probability as was followed to obtain the non-stationary blocking probability in a telephone network [8]. The absorption probability at time $t$,

$$
P_{N+1}(t)=\frac{\alpha(t, 0)^{N+1}}{(N+1) !}-K_{N+1} P_{N+1},
$$

and hence the explicit form of the solution is

$$
P_{N+1}(t)=\frac{\Lambda(t)^{N+1}}{(N+1) !}-K_{N+1} \frac{\Lambda(t)^{N+1}}{(N+1) !}+K_{N+1}^{2} \frac{\Lambda(t)^{N+1}}{(N+1) !}-\cdots,
$$

where

$$
\Lambda(t)=e^{-\mu t} \int_{0}^{t} e^{\mu s} \lambda(s) d s
$$

and $K_{N+1}$ is a Volterra operator defined by the kernel $K_{N+1}(t, \tau)$

$$
=\sum_{j=0}^{N} \frac{\alpha(t, \tau)^{j}}{j !} e^{-(N+1-j) \mu(t-\tau)}\left(\begin{array}{c}
N+1 \\
j+1
\end{array}\right)[\lambda(\tau)-(j+1) \mu],
$$

where $\alpha(t, \tau)=e^{-\mu t} \int_{\tau}^{t} e^{\mu s} \lambda(s) d s$. Note that $\Lambda(t)=\alpha(t, 0)$.

The analytical solution of the absorption probability (7) is complex for an arbitrary arrival rate $\lambda(t)$. Hence, the numerical methods may be advantageously used. The absorption probability can be computed from the linear integral equation (6) which is a Volterra equation of the second kind [9]. Apart from this, we have discussed another method in [5] by solving a set of differential equations with the given initial conditions (5). The differential equations can be obtained from (1)-(4) as the following: Transpose the term $P_{n}(t)$, divide the equation by $h$ and take the limits as $h \rightarrow 0$. Applying this for all $n$, we get

$$
\mathbf{P}^{\prime}(t)=\mathbf{M}(t) \mathbf{P}(t)
$$

where $\mathbf{P}(\mathrm{t})$ is a vector $\left[P_{0}(t) P_{1}(t) \ldots P_{N+1}(t)\right]^{T}$ and $\mathbf{M}(\mathrm{t})$ is a $(N+2) \times(N+2)$ matrix of variable coefficients.

For a general holding time distribution, it is difficult to obtain the analytical solution of the absorption probability. Hence, we use the method of simulation to estimate it in general.

Now, we observe the behaviour of the absorption probability if both the capacity $N$ and the arrival rate $\lambda(t)$ increase but the ratio remains constant. Let us assume the arrival rate at time $t$, $\lambda(t)=\lambda_{0} f(t)$, where $\lambda_{0}$ is the arrival rate at $t=0$. Assume that $\lambda_{0}=r N, r$ is a constant.
As an example, the absorption probabilities of a single link are plotted for different values of $N$ in Figs. 1(a) and 1(b) for exponential and Weibull holding times respectively. We consider a linear growth function $f(t)=1+t / 2$ and the constant ratio $r=0.7$. As an example of the general holding time distribution, we consider a Weibull distribution with the shape parameter $\beta=0.5$ and the scale parameter $\alpha=0.5$. Note that the distribution has mean 1 . Recall that the probability density function of the Weibull distribution is given by

$$
f(x)=\frac{\beta}{\alpha}\left(\frac{x}{\alpha}\right)^{\beta-1} e^{-(x / \alpha)^{\beta}} .
$$

Note that the coefficient of variation(COV) of the Weibull distribution with $\beta=0.5$ is $\sqrt{5}$ which shows a behaviour similar to a hyper-exponential distribution [10].

Observe from Figs. 1(a) and 1(b) that for given $r$ and $f(t)$, the absorption probability becomes more and more steeper as the capacity increases and it becomes almost a step function for a large capacity independent to the holding time distribution. This is true for any arbitrary growth function $f(t)$ and for any ratio $r$. Hence, the absorption probability of a single link with a very large capacity can be approximated by a step function provided the traffic arrival rate is also very large. This behaviour can be explained as follows:

Observe that eqn. (4) is also true for a general holding time distribution. As the arrival process is Poisson with intensity $\lambda(t)$, the transition rate from the transient state $P_{N}(t)$ to the absorption state $P_{N+1}(t)$ is $\lambda(t)$ and it is independent of the holding time distribution as there is no transition from the absorption state. Hence, we can write in general
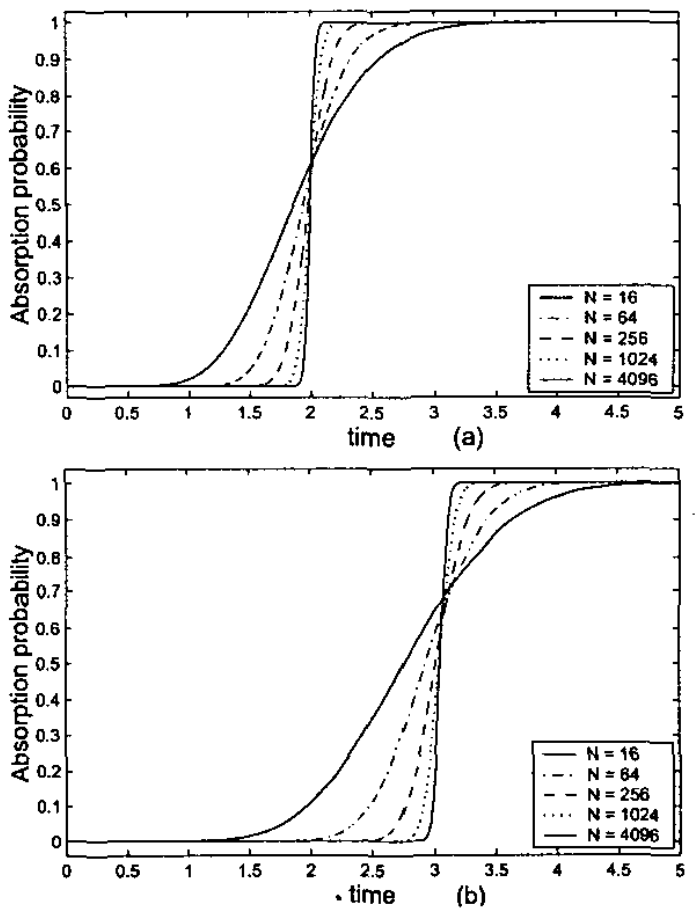

Fig. 1. The absorption probabilities of a single link network for different values of $N$, arrival rate at time $t, \lambda(t)=0.7 N(1+t / 2)$ and mean holding time unity. Holding time distribution: (a) exponential (b) Weibull $(\beta=\alpha=0.5$ ). 


$$
P_{N+1}^{\prime}(t)=\lambda(t) P_{N}(t)
$$

From (11), it follows that $P_{N+1}(t)$ is a monotone nondecreasing function of $t$ with $P_{N+1}(0)=0$. From the initial conditions, $P_{N}(0)=0$. Thus from (11), $P_{N+1}^{\prime}(0)=0$ and the absorption probability $P_{N+1}(t)$ remains zero as long as the slope $P_{N+1}^{\prime}(t)$ remains zero. We shall prove in the next section that as the capacity $N$ and the arrival rate $\lambda(t)$ approach infinity, there exists a $T_{0}$ such that the function $P_{N+1}^{\prime}(t)$ switches from zero to infinity at $t=T_{0}$ which triggers the change of absorption probability from 0 to 1 at $t=T_{0}$. We define $T_{0}$ as the transition time.

\section{ASYMPTOTIC ANALYSIS}

We are interested in finding the transition time $T_{0}$ for a given function of traffic arrival when both the capacity $N$ and the arrival rate $\lambda(t)$ approach infinity while the ratio remains constant. The holding time of a lightpath has a general distribution $G$ with a finite mean. We can model the arrival rate by the following:

$$
\lambda(t)=\lambda_{0} f(t)=r N f(t),
$$

where $r$ is a constant and $f(t)$ is a continuous function of $t$ with $f(0)=1$. We shall derive the explicit expression of the transient state probabilities $P_{n}(t), n=0,1, \ldots, N$ as $N \rightarrow \infty$.

Partition the lightpaths that arrive during $[0, t], Z(t)$, into those that are in progress at $t, Z_{c}(t)$, and those that have departed by $t, Z(t)-Z_{c}(t)$. Clearly, this is a random partition [12]. Hence, it follows immediately that $Z_{c}(t)$ and $Z(t)-Z_{c}(t)$ are independent random variables with

$P\left[Z_{c}(t)=n\right]=\exp \left[-p_{c}(t) \int_{0}^{t} \lambda(\tau) \mathrm{d} \tau\right] \frac{\left[p_{c}(t) \int_{0}^{t} \lambda(\tau) \mathrm{d} \tau\right]^{n}}{n !}$,

where $p_{c}(t)$ is the probability that a lightpath is in progress at time $t$. Now, we obtain the probability $p_{c}(t)$.

The arrival process $Z(t)$ is Poisson with intensity $\lambda(t)$. Hence, for any $u \in[0, t]$,

$$
P[Z(u)=1 \mid Z(t)=1]=\frac{\int_{0}^{u} \lambda(\tau) \mathrm{d} \tau}{\int_{0}^{t} \lambda(\tau) \mathrm{d} \tau} .
$$

So, given exactly one arrival during $[0, t]$, the probability that the arrival occurs between $u$ and $u+d u \in[0, t]$ is $\lambda(u) \mathrm{d} u / \int_{0}^{t} \lambda(\tau) \mathrm{d} \tau$. Again, given a lightpath arrives at $u \in[0, t]$, the probability that the lightpath is in progress at $t$ is $G^{c}(t-u)$ where $G^{c}(t)$ is the complementary cumulative distribution function(CCDF) of the lightpath holding time. Hence, by integration over all $u$, the probability that a lightpath is in progress at $t$,

$$
p_{\mathrm{c}}(t)=\frac{\int_{0}^{t} \lambda(u) G^{c}(t-u) \mathrm{d} u}{\int_{0}^{t} \lambda(\tau) \mathrm{d} \tau} .
$$

Thus, from (13) and (15), we get

$$
\begin{aligned}
& P\left[Z_{c}(t)=n\right]=\exp \left[-\int_{0}^{t} \lambda(u) G^{c}(t-u) \mathrm{d} u\right] \frac{\left[\int_{0}^{t} \lambda(u) G^{c}(t-u) \mathrm{d} u\right]^{n}}{n !} \\
& \quad \text { or } P_{n}(t)=e^{-\Lambda_{g}(t)} \frac{\Lambda_{g}(t)}{n !}, \quad n=0,1, \ldots, N, \\
& \text { where } \Lambda_{g}(t)=\int_{0}^{t} \lambda(u) G^{c}(t-u) \mathrm{d} u=N \Omega(t) \\
& \quad \Omega(t)=r \int_{0}^{t} G^{c}(t-u) f(u) \mathrm{d} u=r G^{c}(t) * f(t)
\end{aligned}
$$

Note that for a growing traffic, $f(t)$ is a strictly nondecreasing function of $t$ with $f(0)=1$. So from (18), the derivative of $\Omega(t)$

$$
\begin{aligned}
\Omega^{\prime}(t) & =r G^{c^{\prime}}(t) * f(t)+r G^{c}(0) f(t), \\
& \geq r G^{c^{\prime}}(t) * f(0)+r f(t), \\
& \geq r G^{c}(t)+r f(0) \geq r>0, \quad \text { for all } t \geq 0 .
\end{aligned}
$$

Thus, $\Omega(t)$ is a strictly increasing function of $t$ with $\Omega(0)=0$.

Consider the series $\left\{P_{n}(t)\right\}$. Note that the terms of the series $\left\{P_{n}(t)\right\}$ are positive. Hence we can apply the ratio test for the test of convergence [11]. From (16) and (17),

$$
\frac{P_{n+1}(t)}{P_{n}(t)}=\frac{\Lambda(t)}{n+1} \rightarrow \Omega(t) \text { as } n \rightarrow \infty .
$$

Hence the series is convergent if $\Omega(t)<1$ and divergent if $\Omega(t)>1$. For $\Omega(t)=1$, the series may converge or diverge, we do not have a definite conclusion from the ratio test.

Now, from (11), (12), (16) and (17), the derivative of $P_{N+1}(t)$,

$$
P_{N+1}^{\prime}(t)=r N f(t) e^{-N \Omega(t)} \frac{[N \Omega(t)]}{N !} .
$$

Recall the Stirling 's formula $N ! \sim \sqrt{2 \pi} N^{N+\frac{t}{2}} e^{-N}$ and the ratio of the two sides tends to unity as $N \rightarrow \infty$ [7]. Hence from (20),

$$
P_{N+1}^{\prime}(t)=\frac{1}{\sqrt{2 \pi}} r f(t) \sqrt{N}\left[\frac{\Omega(t)}{e^{\Omega(t)-1}}\right]^{N} .
$$

Note that $\frac{\Omega(t)}{e^{\Omega(t)-1}}$ is a continuous function of $\Omega(t)$ and bounded by 0 and 1 for all $\Omega(t) \geq 0$. It has a unique maximum at $\Omega(t)=1$. If $\Omega(t)=1, P_{N+1}^{\prime}(t)=\frac{1}{\sqrt{2 \pi}} r f(t) \sqrt{N} \rightarrow \infty$ as $N \rightarrow \infty$. If $\Omega(t) \neq 1$, applying the L'Hospital's rule in (21), $P_{N+1}^{\prime}(t) \rightarrow 0$ as $N \rightarrow \infty$. Again, $\Omega(t)$ is a strictly increasing function of $t$ with $\Omega(0)=0$. Hence, the absorption probability

$$
\begin{aligned}
P_{N+1}(t) & \rightarrow 0 \text { if } \Omega(t)<1, \\
& \rightarrow 1 \text { otherwise, as } N \rightarrow \infty .
\end{aligned}
$$

Note that, as $\Omega(t)$ is a monotone increasing function of $t, T_{0}$ is the unique solution of the nonlinear equation $\Omega(t)=1$.

Remark: If $f(t)$ is not a non-decreasing function of $t, \Omega(t)$ is not necessarily a monotone increasing function, but we can still obtain $T_{0}$ as the minimum of the roots of the equation $\Omega(t)=1$.

So, finally we can write, $\Omega\left(T_{0}\right)=1$ and from (17),

$$
\int_{0}^{T_{0}} G^{c}\left(T_{0}-\tau\right) \lambda(\tau) d \tau=G^{c}\left(T_{0}\right) * \lambda\left(T_{0}\right)=N
$$

This can be explained as follows: If a lightpath arrives at $\tau \in$ $\left[0, T_{0}\right]$, the probability that it is still in progress at $T_{0}$ is $G^{c}\left(T_{0}-\right.$ $\tau)$. Again, the number of lightpath arrivals during $\tau$ and $\tau+\mathrm{d} \tau$ is $\lambda(\tau) \mathrm{d} \tau$. Now, integrating over all $\tau \in\left[0, T_{0}\right]$, the number of busy wavelengths at $T_{0}$ is the left hand side of (23) and if these occupy all the wavelengths, the link will be absorbed.

Approximation of absorption probability based on asymptotic results

Observe the eqns. (6) and (9), the computation of exact absorption probability has a large complexity and it increases with capacity $N$. Hence, we propose an approximation for finite $N$ based on the asymptotic results. From (11) and (21), the absorption probability for a finite $N$ can be approximated by

$$
P_{N+1}(t)=\int_{0}^{t} \lambda(\tau) P_{N}(\tau) d \tau \approx \frac{1}{\sqrt{2 \pi} \bar{N}} \int_{0}^{t} \lambda(\tau)\left[\frac{\Omega(\tau)}{e^{\Omega(\tau)-1}}\right]^{N} d \tau
$$


Note that the complexity of the approximation is low and it can be applied for a general holding time distribution. As an example, the exact and approximate absorption probabilities for a linear traffic growth are plotted as functions of time in Fig. 2 where the absorption probabilities are in a logarithmic scale. Observe that the approximations are very close to the exact values for low absorption probabilities and it provides an upper bound on the exact absorption probability. Again, note from (16) that $P_{N}(t)$ is a monotone increasing function of $t$ provided $\Omega(t)<\mathrm{l}$. Thus, the absorption probability at time $t \in\left(0, T_{0}\right)$,

$$
P_{N+1}(t)<P_{N}(t) \int_{0}^{t} \lambda(\tau) d \tau .
$$

Thus, if $P_{N}(t)$ is equal to $p_{t h} / \int_{0}^{t} \lambda(\tau) d \tau$, the exact absorption probability at time $t, P_{N+1}(t)$ will be bounded by $p_{t h}$. Again from $(16), P_{N}(t)=p_{t h} / \int_{0}^{t} \lambda(\tau) d \tau$ if

$$
\begin{aligned}
& \Lambda_{g}(t)-N \log \Lambda_{g}(t)+(N-0.5) \log N-(N+\gamma)=0, \\
& \text { or } N \approx \exp \left[1+W\left(\frac{\gamma-\Lambda_{g}(t)}{e \Lambda_{g}(t)}\right)\right] \Lambda_{g}(t)
\end{aligned}
$$

where $\gamma=-\log \left(\sqrt{2 \pi} p_{t h} / \int_{0}^{t} \lambda(\tau) d \tau\right)$ and $W(z)$ is the Lambert's function i.e., $W(z)$ is the solution of the equation $w e^{w}=$ $z$. Note that (27) provides an explicit solution, however, (26) is as easy to solve and is the preferred method. Note that for a large $\Lambda_{g}(t),\left(\gamma-\Lambda_{g}(t)\right) /\left(e \Lambda_{g}(t)\right) \approx-1 / e$ and $W(-1 / e)=-1$. So for large traffic, $N \approx \Lambda_{g}(t)$ which is similar to (23).

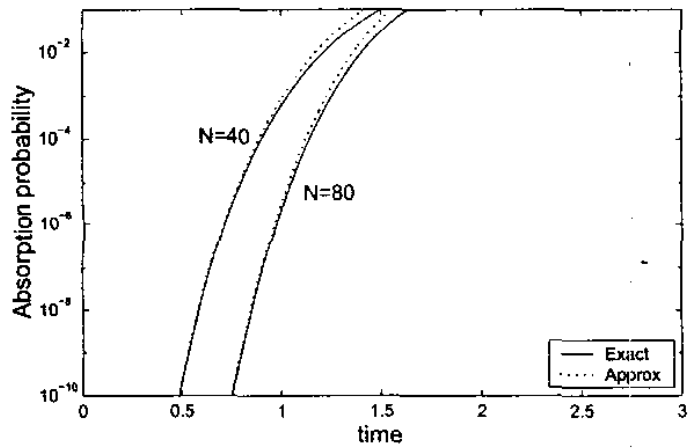

Fig. 2. Comparison of exact and approximate absorption probabilities for single link network. Arrival rate $\lambda(t)=0.7 N(1+t / 2)$ and holding time distribution is exponential with mean unity.

\section{ROUTING AND DIMENSIONING OF A LARGE NETWORK}

We now describe the method of routing and dimensioning for a large optical network with the following assumptions.

\section{A. Assumptions regarding the network and traffic}

1) The network consists of finitely many links, connected in an arbitrary fashion. Label the links as $j=1,2, \cdots, J$, and the capacity of link $j$ is $C_{j}$. Define $Q_{j}$ as the cost to set up a single wavelength on link $j$.

2) A route $r$ is a subset of links from $\{1,2, \cdots, J\}$. A lightpath in route $r$ uses $A_{j r}$ wavelengths from link $j$, $A_{j r} \in\{0,1\}$. Denote $\mathbf{R}$ for the set of routes.

3) Lightpaths requesting route $r$ arrive as a time-varying Poisson process with rate $\lambda_{r}(t)$ at time $t$ and hold for a random time with a general distribution $G$.
The assumption that all the links are independent with the sum of arrival rates $\sum_{r \in \mathbf{R}} A_{j r} \lambda_{r}(t)$ as the arrival rate on link $j$ will yield an upper bound on absorption probability [5]. Note that as the arrival processes in all the routes are Poisson, the sum of the arrival processes on a link is also Poisson. Hence, the absorption probability of the $j$-th link at time $t$,

$$
P_{a b s}^{j}(t) \leq F\left(\sum_{r \in \mathbf{R}} A_{j r} \lambda_{r}(t), G, C_{j}\right), \quad j=1,2, \cdots, J,
$$

where $F(\lambda(t), G, N)$ is the absorption probability at time $t$ for a single link of capacity $N$ with time-dependent Poisson arrival rate $\lambda(t)$ and general holding time distribution $G$.

This can be extended for a route, that the absorption probability of a route $r \in \mathbf{R}$ at time $t$,

$$
L_{r}(t) \leq 1-\prod_{j \in r}\left[1-F\left(\sum_{r \in \mathbf{R}} A_{j r} \lambda_{r}(t), G, C_{j}\right)\right]
$$

But the upper bounds are close to the exact values for low absorption probabilities [5]. In this paper, we consider a network with very low absorption probabilities. So, we can assume that the links are independent and the arrival rate on link $j$ is equal to $\sum_{r \in \mathbf{R}} A_{j r} \lambda_{r}(t)$.

The aim of this paper is to route the traffic in appropriate paths and determine the link capacities of a large optical network so that it requires no upgradation till a given time $T$.

If the traffic is heavy, the required capacities will be large and we can apply the asymptotic analysis on all the links with $T=T_{0}$. For a given traffic matrix, the arrival rate on link $j$ is $\sum_{r \in \mathbf{R}} A_{j r} \lambda_{r}(t)$. The link can accommodate all the arrivals during $(0, T)$, if the absorption probability of link $j$ remains zero until $T$. Hence, from (23), the capacity required for link $j$,

$$
C_{j}=\sum_{r \in \mathbb{R}} A_{j r} \int_{0}^{T} G^{c}(T-\tau) \lambda_{r}(\tau) d \tau .
$$

Hence, the cost to deploy the capacities in all the links of the network is

$$
\sum_{j=1}^{J} Q_{j} C_{j}=\sum_{r \in \mathbf{R}} \int_{0}^{T} G^{c}(T-\tau) \lambda_{r}(\tau) d \tau \sum_{j=1}^{J} Q_{j} A_{j r} .
$$

The term $\int_{0}^{T} G^{c}(T-\tau) \lambda_{r}(t) d \tau$ is constant for a given $T$ as the holding time distribution $G$ and the traffic arrival rate $\lambda_{r}(t)$ are known. Now we shall find the optimum routing so as to minimize the linear cost. The cost will be minimum if the terms $\sum_{j=1}^{J} Q_{j} A_{j r}$ are minimum for every $r \in \mathbf{R}$ which determines the route. Hence, if we select the least cost path for the traffic between a pair of nodes, the total cost $\sum_{j=1}^{J} Q_{j} C_{j}$ will be minimum. Hence, if we assume fixed routing, the least cost paths are the optimum routes. Alternative routing or any other routing schemes would not make any improvement because it is guaranteed that there is a path available in the direct route for $0 \leq t<T$ and all the links are expected to be absorbed for $t>T$.

Note that the proposed method of routing and dimensioning is easy and computationally very efficient. But it is useful if the traffic arrival rates and hence the computed capacities on all the links are fairly large. However, it may not be the case in practice because the flow of traffic through all the links are not uniform and the traffic on some links may be low. Observe the Figs. 1(a) and 1(b), the absorption probability for a moderate value of $N$ 
at the asymptotic transition time $T_{0}$ is high ( 0.6 or more) and the capacity required to keep the absorption probability below a threshold probability $(0.02$ or less) in a given time period $(0, T)$ is greater than the value estimated by (29). Hence for a light traffic, the method proposed is optimistic, however, it provides good lower bounds on the capacities.

For a general range of traffic, the link capacities can be calculated from (24) so that the absorption probability on link $j$ is bounded by a given threshold probability $p_{t h}^{j}$ in the time period $(0, T)$. Again, the absorption probability on the $j$-th link will be bounded by $p_{t h}^{j}$ in $(0, T)$ if the capacity is calculated by $(26)$ and it provides upper bounds on the link capacities. For any range of traffic, if the threshold probabilities are small, the computed capacities will be close to the upper bounds. On the other hand, if the threshold probabilities are large, the capacities can be approximated by the lower bounds. Note that, for a large traffic, both the upper and lower bounds will converge.

\section{RESULTS AND DISCUSSION}

We consider the ARPANET (shown in Fig. 3) as an arbitrary network and solve the routing and dimensioning problem for a moderate rate of traffic arrivals. Note that the network with the given costs is equivalent to a weighted graph. Routes are selected as the least cost paths between a pair of nodes. This can be obtained by applying the Dijkstra's algorithm for each node. If there are multiple least cost paths, we consider any one of them because all the paths have equal contributions towards the total cost. The total number of possible routes is 190 , the maximum possible hop length is 6 and the numbers of routes with 1 , $2,3,4,5$ and 6 hops are $32,51,56,32,18$ and 1 respectively.

For the network traffic, we assume identical traffic arrival rates between every pair of nodes. Hence, there is an uniform traffic in all the routes. We assume a linear traffic growth and the traffic arrival rate on route $r \in \mathbf{R}$ at time $t, \lambda_{r}(t)=\lambda_{r 0}(1+t / 2)$ where $\lambda_{r 0}$ is the initial arrival rate at $t=0$. Here we consider $\lambda_{r 0}=2$ for all $r$. For an exponential holding time distribution, we calculate the link capacities by both the exact (from (9)) and approximation (from (24)) methods. We also calculate the asymptotic lower bounds from (29) and the upper bounds from (26) and the results are presented in Table 1. As an example of a general holding time distribution, we consider the Weibull distribution with shape parameters $\beta=0.5$ and $\beta=2$ which show the hyper- and hypo-exponential behaviours respectively. $\alpha$ 's are chosen so that the means become unity which results $\alpha=0.5$ and 1.1284 respectively. The lower bounds, the upper bounds and the capacities estimated by the approximation method are shown in Table I. In all the cases, we consider that

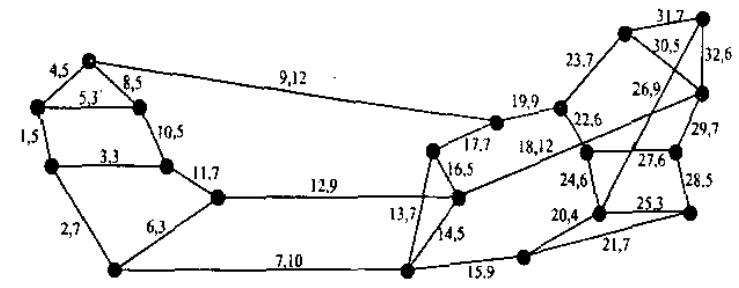

Fig. 3. A 20 node, 32 link network representing a skeleton of the original Arpanet. The numbers on the links represent the link numbers and the costs to set up unit capacity on those links.
TABLE I

CAPACITIES ALLOTTED TO DIFFERENT LINKS OF THE ARPA NETWORK

\begin{tabular}{|c|c|c|c|c|c|c|c|c|c|c|}
\hline \multirow{4}{*}{$\begin{array}{l}\text { Link } \\
\text { No }\end{array}$} & \multicolumn{10}{|c|}{ Holding Time Distribution } \\
\hline & \multicolumn{4}{|c|}{ Exponential } & \multicolumn{6}{|c|}{ Weibult $(\alpha, \beta)$} \\
\hline & & & $E 1$ & & & 5,0 & & & 284 & \\
\hline & LB & Ex & Ap & UB & LBB & Ap & UB & LB & Ap & UB \\
\hline 1 & 38 & 55 & 57 & 61 & 26 & $42]$ & 46 & 44 & 64 & 69 \\
\hline 2 & 58 & 79 & 81 & 87 & 39 & 59 & 64 & 67 & 93 & 99 \\
\hline$\sqrt{3}$ & 35 & 52 & 53 & 57 & 24 & 39 & 43 & 41 & 60 & 65 \\
\hline 4 & 35 & 52 & 53 & 57 & 24 & 39 & 43 & 41 & 60 & 65 \\
\hline 5 & 23 & 37 & 38 & 42 & 16 & 29 & 31 & 27 & 43 & 47 \\
\hline 6 & 38 & 55 & 57 & 61 & 26 & $\overline{42}$ & 46 & 44 & 64 & 69 \\
\hline 7 & 86 & 112 & 115 & 123 & 59 & 83 & 90 & 101 & 131 & 140 \\
\hline 8 & 38 & 55 & 57 & 61 & 26 & 42 & 46 & 44 & $\overline{64}$ & 69 \\
\hline 9 & 92 & 119 & $|2|$ & $1 \overline{30}$ & 63 & 88 & 94 & 107 & 139 & 148 \\
\hline 10 & 46 & 66 & \begin{tabular}{|l|}
67 \\
\end{tabular} & 72 & 32 & 50 & 54 & 54 & 77 & 82 \\
\hline \begin{tabular}{|l|}
11 \\
\end{tabular} & 66 & 89 & 91 & 98 & 45 & 67 & 72 & \begin{tabular}{|l|}
77 \\
\end{tabular} & 104 & 111 \\
\hline \begin{tabular}{|l|}
12 \\
\end{tabular} & 84 & 109 & 111 & 119 & \begin{tabular}{|l|}
57 \\
\end{tabular} & 81 & 87 & \begin{tabular}{|l|}
97 \\
\end{tabular} & 128 & 136 \\
\hline 13 & 38 & 55 & 57 & 61 & \begin{tabular}{|l|}
26 \\
\end{tabular} & 42 & 46 & 44 & 64 & 69 \\
\hline 14 & 21 & 34 & \begin{tabular}{|l|}
35 \\
\end{tabular} & 37 & 14 & 26 & 28 & 24 & $\overline{39}$ & 42 \\
\hline \begin{tabular}{|l|}
15 \\
\end{tabular} & 101 & 129 & $133 !$ & 140 & 69 & 95 & 102 & 117 & 151 & 160 \\
\hline \begin{tabular}{|l|}
16 \\
\end{tabular} & 35 & 52 & 53 & 57 & 24 & 39 & 43 & 41 & 60 & 65 \\
\hline \begin{tabular}{|l|}
17 \\
\end{tabular} & 52 & 72 & 74 & 80 & 36 & 54 & 59 & 61 & $\overline{85}$ & 90 \\
\hline \begin{tabular}{|l|}
18 \\
\end{tabular} & 84 & 109 & 111 & 119 & \begin{tabular}{|l|}
57 \\
\end{tabular} & 81 & 87 & 97 & 128 & 136 \\
\hline \begin{tabular}{|l|}
19 \\
\end{tabular} & 101 & 129 & 131 & 140 & 69 & 95 & 102 & 117 & 151 & 160 \\
\hline 20 & 38 & 55 & \begin{tabular}{|c|}
57 \\
\end{tabular} & 61 & 26 & 42 & 46 & 44 & 64 & 69 \\
\hline 21 & $5 \overline{5}$ & 76 & 78 & 84 & 37 & 57 & 62 & 64 & 89 & 95 \\
\hline 22 & 61 & 83 & 84 & 91 & 41 & 62 & 67 & 71 & \begin{tabular}{|l|}
96 \\
\end{tabular} & 103 \\
\hline 23 & 38 & 55 & 57 & 61 & 26 & 42 & 46 & 44 & 64 & 69 \\
\hline 24 & 49 & 69 & 71 & 76 & 34 & 52 & 56 & 57 & 81 & $\overline{86}$ \\
\hline 25 & 23 & 37 & 38 & 42 & 16 & 29 & 31 & 27 & 43 & 47 \\
\hline 26 & 21 & 34 & 35 & 37 & 14 & 26 & 28 & 24 & 39 & 42 \\
\hline 27 & 21 & 34 & 35 & 37 & 14 & 26 & 28 & 24 & 39 & 42 \\
\hline 28 & 23 & 37 & 38 & $4 \overline{2}$ & 16 & 29 & 31 & 27 & $4 ?$ & 47 \\
\hline 29 & 41 & 59 & 60 & 65 & 28 & \begin{tabular}{|l|}
45 \\
\end{tabular} & 48 & 47 & 68 & 73 \\
\hline \begin{tabular}{|l|}
30 \\
\end{tabular} & 21 & 34 & 35 & 37 & 14 & 25 & 28 & 24 & 39 & 42 \\
\hline 31 & 38 & $5 \overline{5}$ & 57 & 61 & 26 & 42 & $\overline{46}$ & 44 & 64 & 69 \\
\hline$\overline{32}$ & 26 & 41 & 42 & 46 & 18 & $\overline{32}$ & 34 & 31 & 48 & 51 \\
\hline
\end{tabular}

the absorption probability on all the links are bounded by 0.005 $(0.5 \%)$ ai time $T=2$.

Observe from Table I that for the exponential holding time distribution, the estimated capacities based on the approximation method are close to the exact soiutions. Again, for all the holding time distributions, the upper bounds are close to the estimated capacities; hence, the upper bounds can provide a good solution especially for low absorption probabilities.

\section{REFERENCES}

[1] R. Ramaswami and K. N. Sivarajan, "Optical Networks : A Practical Perspective." Morgan Kaufmann Publishers, San Fransisco, 1998.

[2] A Girard, "Routing and Dimensioning in circuit-swilched networks," Addison-Wesley, 1990.

[3] F. P. Kelly, "Network routing," Philosophical Transactions of the Royal Society, A337, pp. 343-367, 1991

[4] T. K. Nayak and K. N. Sivarajan, "A new approach to dimensioning optical networks," IEEE Journal on Selected Areas in Communications, vol. 20 , No. 1, pp. 134-148, January 2002.

[5] T. K. Nayak and K. N. Sivarajan, "Dimensioning optical networks under traffic growth models," IEEE International Conference on Communica. tions, April 2002.

[6] A. Papoulis, "Probability. Random Variables and Stochastic Processes," 3rd edn. McGraw-Hill, New York, 1991.

[7] W. Feller, "An Introduction to Probability Theory and its Applications." vol. 1, 3rd edn. Wiley, New York, 1968

[8] D. L. Jagerman, "Nonstationary blocking in telephone traffic", Bell System Technical Journal, Vol. 54, No. 3, pp. 625-661, 1975.

[9] V. Volterra, "Theory of Functionals and of Integro-differential Equations." Dover, New-York, 1959.

[10] A. Feldmann and W. Whitt, "Fitting mixtures of exponentials to long-tail distributions to analyze network performance models" IEEE Infocom ' 97 , pp. 1098-1106, 1997.

[11] G. B. Arfken and H. J. Weber, "Mathematical Methods for Physicists", 4th edn. Academíc Press, New York, 1995.

[12] R. W. Wolff, "Stochastic Modeling and the Theory of Quetes", 4th edn. Prentice-Hall, Englewood Cliffs, New Jersey, 1989. 